\title{
(- OPEN ACCESS \\ Can patient-reported measurements of pain be used to improve cancer pain management? A systematic review and meta-analysis
}

\author{
Rosalind Adam, ${ }^{1}$ Christopher D Burton, ${ }^{1}$ Christine M Bond, ${ }^{1}$ \\ Marijn de Bruin, ${ }^{2}$ Peter Murchie ${ }^{1}$
}

- Additional material is published online only. To view please visit the journal online (http://dx.doi.org/10.1136/ bmjspcare-2016-001137).

${ }^{1}$ Centre of Academic Primary Care, University of Aberdeen, Aberdeen, UK

${ }^{2}$ Aberdeen Health Psychology Group, Institute of Applied Health Sciences, University of Aberdeen, Aberdeen, UK

\section{Correspondence to} Dr Rosalind Adam, Centre of Academic Primary Care, University of Aberdeen, Room 1:131, Polwarth Building, Foresterhill, Aberdeen AB25 2ZD, UK; rosalindadam@abdn.ac.uk

Received 2 March 2016 Revised 26 August 2016 Accepted 28 October 2016 Published Online First 22 November 2016

\section{CrossMark}

To cite: Adam $R$, Burton $C D$, Bond $\mathrm{CM}$, et al. BMJ

Supportive \& Palliative Care 2017;7:373-382.

\section{ABSTRACT}

Purpose Cancer pain is a distressing and complex experience. It is feasible that the systematic collection and feedback of patientreported outcome measurements (PROMs) relating to pain could enhance cancer pain management. We aimed to conduct a systematic review of interventions in which patient-reported pain data were collected and fed back to patients and/or professionals in order to improve cancer pain control.

Methods MEDLINE, EMBASE and CINAHL databases were searched for randomised and non-randomised controlled trials in which patient-reported data were collected and fed back with the intention of improving pain management by adult patients or professionals. We conducted a narrative synthesis. We also conducted a meta-analysis of studies reporting pain intensity.

Results 29 reports from 22 trials of 20 interventions were included. PROM measures were used to alert physicians to poorly controlled pain, to target pain education and to link treatment to management algorithms. Few interventions were underpinned by explicit behavioural theories. Interventions were inconsistently applied or infrequently led to changes in treatment. Narrative synthesis suggested that feedback of PROM data tended to increase discussions between patients and professionals about pain and/or symptoms overall. Meta-analysis of 12 studies showed a reduction in average pain intensity in intervention group participants compared with controls (mean difference $=-0.59(95 \% \mathrm{Cl}-0.87$ to $-0.30)$ ).

Conclusions Interventions that assess and feedback cancer pain data to patients and/or professionals have so far led to modest reductions in cancer pain intensity. Suggestions are given to inform and enhance future PROM feedback interventions.

\section{INTRODUCTION}

Pain is the most frequent complication of cancer. ${ }^{1}$ Approximately $40 \%$ of patients experience moderate-to-severe pain at diagnosis, rising to $70 \%$ at the end of life. ${ }^{1}$ Cancer pain control is frequently suboptimal, despite effective treatments being available. ${ }^{2}$ Under-reporting of pain by patients, inadequate communication about pain between patients and healthcare professionals, and inadequate assessment of pain by professionals are known to contribute to poor pain control. ${ }^{34}$

Traditional clinical consultation models rely on a question and answer-based dialogue between the patient and professional during which patients are prompted to report and describe problems. This may underestimate pain for several reasons. Retrospective reports by patients are subject to recall bias, underestimation and imprecision. ${ }^{5}$ Patients may fail to report cancer pain if they expect that pain is an inevitable consequence of cancer, if they believe that pain is a useful indicator of disease activity, or if they fear that symptom discussions will shift the professional's focus away from the treatment of disease. ${ }^{6}$ Pain can be a complex and subjective experience, and patients can have difficulties judging the validity of pain as a presenting symptom that warrants medical attention. ${ }^{7}$ Professionals may not ask about or adequately assess the details of the patient's pain. ${ }^{8}$ Therefore, it is possible that the traditional consultation model could lead to specific deficiencies in cancer pain management. 
The potential value of collecting patient-reported outcome measurements (PROMs) is increasingly being recognised in clinical practice. ${ }^{9}$ PROMs are defined as: 'measurements of any aspect of a patient's health status that come directly from the patient, without interpretation of the patient's response by a clinician or anyone else'. ${ }^{10}$ Patient-reported outcomes might be collected from patients via interviews, questionnaires or diaries. Recently, digital technology has enabled PROMs to be collected remotely via hand-held devices and web-based forms. It has been suggested that PROMs might have value in the provision of patient health status information to clinicians; monitoring response to treatments (and their side effects); detecting unrecognised problems; and improving health management behaviours by patients and professionals. ${ }^{11}$ In oncology, PROMs have been shown to improve patient satisfaction with their care and to increase the frequency of discussion of patient outcomes during consultations. ${ }^{12} 13$

Despite the impact of pain on the well-being of patients with cancer and the potential value of using PROMs to enhance cancer pain management, it is currently unclear whether PROM interventions can have an impact on patient pain outcomes. This review aims to synthesise the evidence on interventions which have used patient-reported pain measurements to enhance the management of cancer-related pain by making these pain data available to patients and/or healthcare providers; to describe the interventions and their main components; and to determine whether the systematic collection of patient reported pain data can improve cancer pain outcomes.

\section{METHODS}

A systematic review was conducted to identify randomised controlled trials (RCTs) and controlled trials of interventions which involved the systematic collection of patient-reported measurements of pain related to cancer or its treatment. The review was conducted according to 'the Preferred Reporting Items for Systematic reviews and Meta-Analyses' (PRISMA) criteria. A review protocol was registered and is available at: http://www.crd.york.ac.uk/PROSPEROFILES/15217_ PROTOCOL_20141027.pdf

\section{Inclusion and exclusion criteria}

This review considered RCT and non-RCT in which patient-reported measurements of pain were collected and fed back to patients and/or clinicians with the intention of improving cancer pain management behaviours by adult patients or professionals. It was judged that non-randomised studies were relevant to the assessment of PROM intervention components. Inclusion and exclusion criteria are summarised in table 1.

\section{Search strategy}

There were three groups of search terms relating to: cancer pain; self-report and measurement; and
Table 1 Summary of inclusion and exclusion criteria

Inclusion criteria Exclusion criteria

RCTs and controlled intervention trials. All comparators considered

Adults aged 18 years and over

Non-malignant pain

All cancer types, grades, stages and prognoses

Cancer survivors without active disease

Pain outcomes reported only within composite measures of quality of life or distress scores

Participants experiencing pain

relating to cancer or its treatment

(including anticancer therapies and

surgical procedures) at enrolment, or

who were considered to be at risk

of such pain during the intervention

period

Intervention includes systematic

collection of patient-reported pain

data, alone or in combination with

data on other symptoms or

outcomes

$\mathrm{RCT}$, randomised controlled trial.

behavioural change relating to pain management. Keywords and Boolean operators were explored and combined on the advice of a senior medical librarian to search MEDLINE, EMBASE and CINAHL databases from inception. Database searches took place in November and December 2014 and a MEDLINE search was updated in December 2015. Detailed search strategies and dates are shown in online supplementary appendix 1 . Reference lists of two reviews of PROMs in oncology ${ }^{12}{ }^{13}$ and all relevant full-text papers included in this review were searched for additional relevant titles.

\section{Study selection}

Study titles and then abstracts of relevant titles were screened independently by two authors (RA and $\mathrm{CMB}$ ). Full texts were retrieved for all unique abstracts which were felt to be potentially relevant by either author, and these were reviewed independently against the inclusion and exclusion criteria by two authors (RA and one of $\mathrm{CMB}, \mathrm{CDB}, \mathrm{PM}$ and $\mathrm{MdB}$ ). Any disagreement was resolved by discussion.

\section{Risk of bias assessment}

Risk of bias was assessed independently by two authors (RA and CDB) according to the Cochrane collaboration risk of bias tool ${ }^{14}$ and inter-rater reliability was assessed using Cohen's $\kappa$ statistic, ${ }^{15}$ calculated on Stata statistical software V.14.

\section{Data extraction and synthesis}

Data extraction was based on the Template for Intervention Description and Replication (TIDieR) checklist. ${ }^{16}$ Study authors were contacted by email where methodological or outcome data were missing from papers. 
As specified in the protocol, we anticipated heterogeneity in interventions and reported outcomes and so carried out a narrative synthesis of the included studies. For those studies which reported outcomes for pain intensity using similar measures, we also conducted a meta-analysis. RevMan V.5 was used for statistical analysis, with a random-effects model in view of the clinical heterogeneity of studies.

\section{RESULTS}

A PRISMA diagram is shown in figure 1. In total, 3412 titles were identified by searching four databases and by screening reference lists. No new studies were identified in the updated MEDLINE search (December 2015); however, one new article was identified after the initial database searches ${ }^{17}$ which was linked to the research team of an earlier study. ${ }^{18}$ Forty-five full-text articles were assessed, of which 29 satisfied the inclusion and exclusion criteria and were included in the narrative synthesis.

\section{Characteristics of the included studies}

There were 29 reports $^{17-45}$ of 22 unique trials of 20 interventions. Twenty trials were RCTs, and two were controlled trials. ${ }^{19} 23$ The trials were published between 1997 and 2015 and were conducted in the USA, the Netherlands, Norway, Canada, Germany and the UK (table 2). There were 5234 unique trial participants. Most studies were conducted in an oncology outpatient setting in patients with mixed cancer types (table 2).

\section{Risk of bias in included studies}

A Cochrane risk of bias summary assessment is shown in table 3. Inter-rater reliability for risk of bias assessment ( $\kappa)$ between the two reviewers was 0.84 (95\% CI 0.75 to 0.88 ), suggesting high levels of agreement. The 'blinding of participants and personnel' category has been omitted from the summary assessment because the nature of the interventions meant that none of the included studies could have blinded the research participants. Only Wilkie et $a l^{45}$ blinded

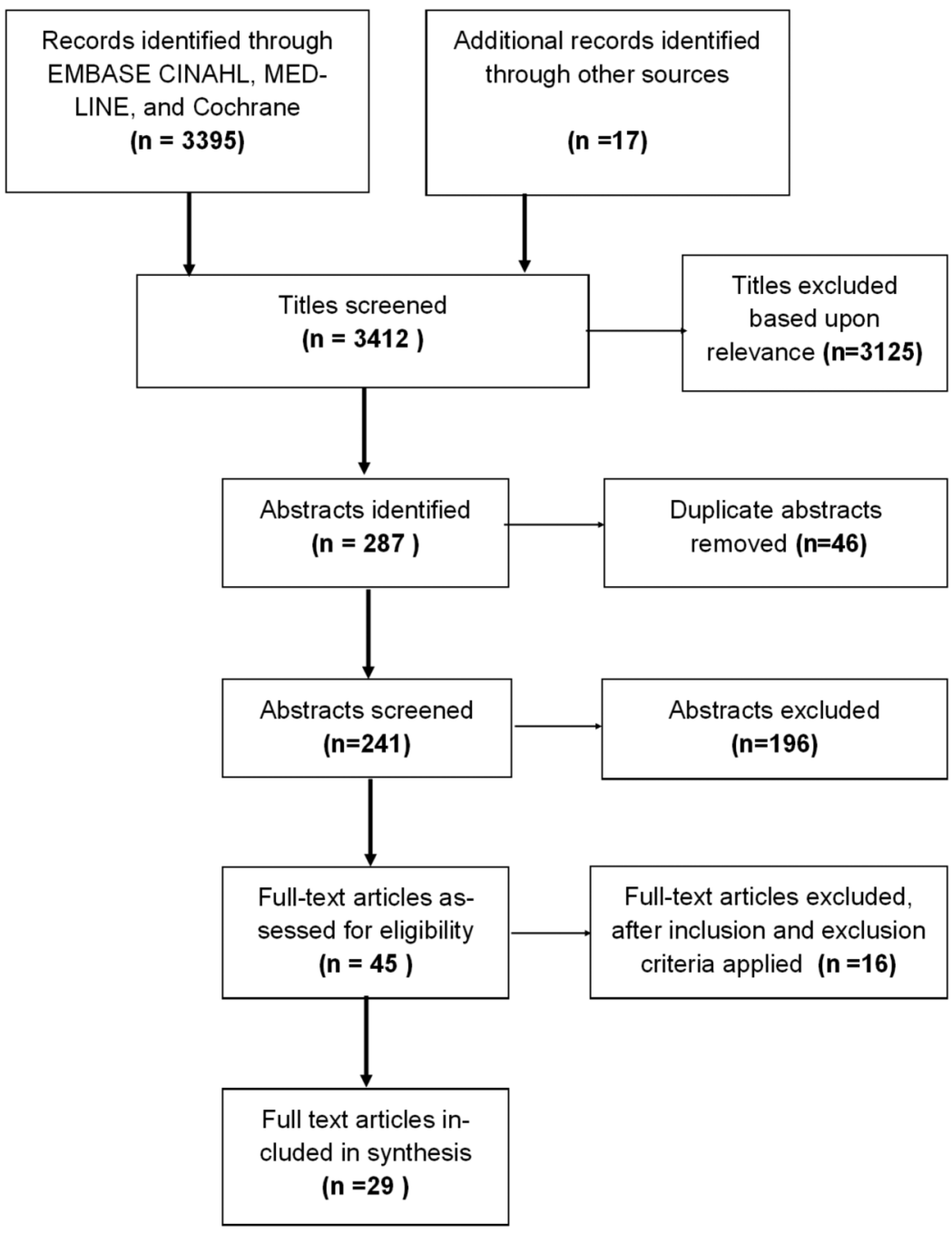

Figure 1 PRISMA chart detailing study identification and selection process. PRISMA, Preferred Reporting Items for Systematic reviews and Meta-Analyses. 
Author, publication year, country, number of participants (n).

Clinical setting

Anderson 2015, ${ }^{17}$ USA, $\mathrm{n}=60$ Outpatient oncology. Breast cancer

Aubin $2006,{ }^{19}$ Canada, $\mathrm{n}=80$

Community palliative care. Mixed cancer types

Berry 2011, ${ }^{20}$ USA, $\mathrm{n}=660$

(ESRA-C 1 intervention)

Berry $2014,{ }^{21}$ USA, $\mathrm{n}=752$

(ESRA-C 2 intervention)

Bertsche $2009{ }^{23}$ Germany, $\mathrm{n}=100$

Cleeland 2011, ${ }^{18}$ USA, $n=100$

De Wit 2001, ${ }^{24}$ the

Netherlands, $n=313$, and Van

Der Peet, ${ }^{44}$ 2009, the

Netherlands, $n=120$

Du Pen $1999,{ }^{26}$ USA, $n=81$

Given $2004,{ }^{27}$ USA, $\mathrm{n}=237$

Hoekstra 2006, ${ }^{28}$ the

Netherlands, $n=146$

Kravitz 2011, ${ }^{29-32}$ USA, $\mathrm{n}=307$

Kroenke $2010,{ }^{33}$ USA, $\mathrm{n}=405$

Miaskowski 2004, ${ }^{34}$ USA, $\mathrm{n}=174$ and Rustoen 2014, ${ }^{39}$ Norway, $n=179$ (PRO-SELF intervention

Mooney $2014,{ }^{35}$ USA, $n=250$

Post $2013,{ }^{36}$ USA, $n=50$

Ruland 2010, ${ }^{37}$ Norway $n=145$ (CHOICE ITPA intervention) Trowbridge 1997, ${ }^{40}$ USA, $n=510$
Outpatient oncology. Mixed cancer types

Outpatient oncology. mixed cancer types

Inpatient oncology. Mixed cancer types

Postoperative outpatient. Primary

lung cancer or lung metastases

Community palliative care. Mixed cancer types

Outpatient oncology. Mixed cancer types

Outpatient oncology. Mixed cancer types

Outpatient oncology. Breast cancer

Outpatient oncology and palliative care. Recurrent or metastatic lung, breast, and upper gastrointestinal cancers

Outpatient oncology. Mixed cancer types

Outpatient oncology. Cancer with bony metastases

Outpatient oncology. Mixed cancer types

Outpatient oncology. Breast cancer

Inpatient and outpatient oncology. Haematological malignancies

Outpatient oncology. Recurrent or metastatic cancer
Monitoring

PROM feedback mechanism (intervention group)

Automated telephone monitoring Oncologist emailed if symptom reached twice weekly for 8 weeks

thresholds. Symptom summaries given to oncologists before scheduled appointments

Twice daily paper diary for 4 weeks

Patient instructed to contact their nurse if pain or analgesic use reached a set threshold. Nurse liaised with prescribing physician

Preclinic on touch screen notebook computers on 2 occasions

Colour graphical summaries handed to the clinician before appointments or attached to clinical notes

Internet-based form (completed at Symptoms above a threshold automatically home or on clinic P(s) at 3 points over 8 weeks

Daily inpatient assessment

Twice weekly automated telephone calls for 4 weeks

Twice daily paper pain diary for 2 months

Daily paper diary for 3 months

Fortnightly report to nurse (face-to-face and by telephone) over 20 weeks

Weekly ratings in a paper booklet

Questionnaire administered by telephone by a health educator on a single occasion prior to a clinic appointment

Automated telephone or online, twice weekly to monthly over 12 months

Daily paper diary for 6 weeks

Daily automated telephone assessment for 45 days

Weekly on a PDA over 160 days.

Preclinic assessments and daily during inpatient admissions over 1 year

Questionnaire immediately before a clinic appointment produced tailored coaching messages on how to describe the problem to the clinical team. PROM graphs and coaching messages could be viewed by the patient at any time

Pain scores linked to algorithmic pain management instructions

An email alert was sent to the advanced nurse practitioner if any symptoms were above a threshold.

Patient's knowledge, attitude and pain ratings used to tailor education and advice about non-pharmacological strategies

Pain ratings, side effects and analgesic use mapped to algorithmic pain management guidelines for physicians

Symptoms above a threshold lead the nurse to provide specific self-management instructions and coaching

Patients were asked to bring the symptom monitor booklet to all clinical appointments.

Health educator met with patients an hou before clinic appointments and used their PROM data to provide tailored pain education, correcting misconceptions, teaching self-management strategies and how to communicate with the physician.

Nurse reviewed symptom reports, liaised with the patient's oncologist and contacted the patient with treatment recommendations. PROM data used to tailor education and coaching. Patients taught to use a weekly pill box, and to use a specific script to communicate with their physician about unrelieved pain and the need for a change in their medication.

Automated alerts faxed or emailed to the patient's oncologist or nurse if symptoms or trends in symptoms reached a threshold.

Patients asked to view videos on the PDA about how to communicate about symptoms and to bring the PDA to clinic appointments. Professionals viewed symptom summaries on the PDA and a printed output was added to clinic notes.

Symptom summaries printed and added to clinical notes to be reviewed by the treating physician

Summary sheet provided to oncologist before the appointment 
Author, publication year, country, number of participants (n).

Clinical setting

Outpatient radiation oncology.

Mixed cancer types

Velikova $2004,{ }^{42}$ UK, $\mathrm{n}=286$

Outpatient oncology. Mixed cancer types

Wilkie $2010,{ }^{45}$ USA, $\mathrm{n}=215 \quad$ Outpatient oncology. Lung cancer
Touch screen questionnaires in the waiting room before appointments for 6 months

Greased pencil on a laminated pain tool on a daily basis
PROM feedback mechanism (intervention group)

Participants asked to bring their diary to scheduled clinic appointments. Participants asked to seek medical attention if pain intensity scores or analgesic use reached a predetermined threshold

Specific symptoms and functional outcomes were displayed individually and tracked longitudinally on graphs provided to the patient's physician.

Patients watched a video on how to monitor and report changes in pain, and encouraged to summarise their pain ratings in note form to help them verbally report pain at scheduled appointments.

PDA, personal digital assistant; PROM, patient-reported outcome measurement.

Table 3 Risk of bias for the included studies

\begin{tabular}{|c|c|c|c|c|c|c|}
\hline & $\begin{array}{l}\text { Random sequence } \\
\text { generation }\end{array}$ & $\begin{array}{l}\text { Allocation } \\
\text { concealment }\end{array}$ & $\begin{array}{l}\text { Blinding of outcome } \\
\text { assessment }\end{array}$ & $\begin{array}{l}\text { Incomplete } \\
\text { outcome data }\end{array}$ & $\begin{array}{l}\text { Selective } \\
\text { reporting }\end{array}$ & $\begin{array}{l}\text { Other } \\
\text { bias }\end{array}$ \\
\hline Anderson 2015 & Yes & Unclear & Unclear & Yes & Yes & Yes \\
\hline Aubin 2006 & Unclear & Unclear & Unclear & Yes & Unclear & No \\
\hline Berry 2011 & Yes & Yes & Unclear & Yes & Yes & Yes \\
\hline Berry 2014 & Yes & Yes & Unclear & Yes & Yes & Yes \\
\hline Bertsche 2009 & No & No & No & Yes & Yes & No \\
\hline Cleeland 2011 & Yes & Yes & No & Yes & Yes & Yes \\
\hline De Wit 2001 & Unclear & Unclear & Unclear & Yes & Yes & Unclear \\
\hline Du Pen 1999 & Yes & Unclear & Yes & Unclear & Yes & Yes \\
\hline Given 2004 & Yes & Yes & Yes & Yes & Yes & Yes \\
\hline Hoekstra 2006 & Unclear & Unclear & Unclear & Unclear & Yes & Unclear \\
\hline Kravitz 2011 & Yes & Yes & Unclear & Yes & Yes & Yes \\
\hline Kroenke 2010 & Yes & Unclear & Yes & Yes & Yes & Yes \\
\hline $\begin{array}{l}\text { Miaskowski } \\
2004\end{array}$ & Unclear & Unclear & No & Yes & Yes & Unclear \\
\hline Mooney 2013 & Yes & Yes & Yes & Yes & Yes & Yes \\
\hline Post 2013 & Unclear & Unclear & Unclear & Yes & Yes & Yes \\
\hline Ruland 2010 & Yes & Yes & Yes & Yes & Yes & Yes \\
\hline Rustoen 2012 & Yes & Unclear & Unclear & Yes & Yes & Unclear \\
\hline $\begin{array}{l}\text { Trowbridge } \\
1997\end{array}$ & Unclear & Unclear & No & Unclear & Unclear & Unclear \\
\hline Vallières 2006 & Unclear & Unclear & No & Unclear & Unclear & No \\
\hline $\begin{array}{l}\text { Van der Peet } \\
2009\end{array}$ & Yes & Unclear & Unclear & Yes & Yes & Unclear \\
\hline Velikova 2004 & Yes & Yes & Yes & Yes & Yes & Yes \\
\hline Wilkie 2010 & Yes & Yes & Yes & Yes & Yes & Yes \\
\hline
\end{tabular}

treating physicians and instructed patient's not to take their pain tools to clinic appointments; however, the remainder of studies expected physicians to act on patient-reported data, and therefore treating physicians tended not to be blinded. In some studies controls also monitored symptoms without feedback to clinicians, and in the remainder controls received usual care without additional pain monitoring.
The results of four studies should be interpreted with caution. Aubin et $a l^{19}$ conducted a nonrandomised study which had high dropouts due to death and hospital admission. The study by Bertsche $e t a^{23}$ was also a non-randomised trial. Methodological details were lacking in the studies by Trowbridge et $a l^{40}$ and Vallières et $a l^{41}$ and the risk of bias in these studies was unclear. 


\section{Theory, rationale and intervention components}

The interventions and their components are summarised in table 2 . Wilkie et $a l^{45}$ based their coaching intervention on Johnson's ${ }^{46}$ behavioural system model for nursing practice. No other interventions used a specific behavioural theory to guide development, although several trials ${ }^{29} 3439$ used self-efficacy and academic detailing theories to inform their interventions.

\section{PROM data collection}

A variety of formats were used to allow patients to report pain and other symptoms. Nine trials used pen and paper, ${ }^{19} \quad 23 \quad 25 \quad 26 \quad 28 \quad 34 \quad 40 \quad 45$ four used touch screen devices or personal digital assistants to collect the data, 20363741 three used automated telephone monitoring, ${ }^{17} 18 \quad 35$ one used web-based systems, ${ }^{21}$ and in two trials, the patient was interviewed by a nurse $^{27}$ or a health educator ${ }^{29}$ for the data. One study offered a choice between automated telephone monitoring or online monitoring. ${ }^{33}$

Pain and symptom monitoring took place immediately before planned outpatient visits in five studies without the option of home symptom monitoring, ${ }^{20} 29374042$ and one study ${ }^{23}$ collected PROMs during an inpatient stay. The remaining studies offered the ability to monitor symptoms at home as required, or at set intervals ranging from twice daily to monthly.

Eight out of 22 studies focused on pain and analgesic monitoring alone and the remainder involved other PROM measures such as mood, quality of life, distress, and analgesic usage. Pain was often monitored alongside other physical symptoms including: nausea, vomiting, constipation, diarrhoea, fatigue, appetite loss, sleep disturbance, cough, breathlessness, fever and dry mouth.

\section{PROM data usage and feedback mechanisms}

The patient-reported outcome data were used in a variety of ways. Summary data were given to a clinician in advance of a consultation in eight studies. ${ }^{1720212836374042}$ None of the clinicians in these studies were given specific instructions about how to use the data except in the study by Vallières et $a l,{ }^{41}$ in which clinicians were asked to alter analgesics according to the WHO's analgesic ladder.

Five studies ${ }^{17} 21272934$ used the patient-generated data to target education on analgesic use, selfmanagement skills and communicating about pain. Berry et $a l^{21}$ embedded automated tailored coaching messages into their web-based intervention. The coaching messages typically focused on how to communicate about unrelieved symptoms with professionals. Four interventions ${ }^{17} \quad 18 \quad 27 \quad 35$ contained automatic alerts to physicians based on predetermined symptom thresholds. One study ${ }^{19}$ also used a symptom threshold concept within their paper diary intervention, instructing patients to contact their nurse if pain intensity or analgesic use crossed a threshold. Four studies ${ }^{23} 26 \quad 27 \quad 33$ linked patientreported data to specific management algorithms to support clinical decision-making.

\section{Intervention fidelity}

Several interventions were not delivered as designed. Mooney et $a l^{35}$ reported that only 20 of 167 (12\%) automated alerts to physicians of symptoms exceeding a threshold resulted in a provider-initiated unscheduled contact. Hoekstra et $a l^{28}$ reported that despite patients being advised to take their symptom monitor to all medical appointments, it was used in only 232 of 1291 (18\%) consultations. Van der Peet et $a l^{44}$ found that 22 of $37(59 \%)$ written recommendations to physicians advising medication changes were ignored. In comparison, one study by Bertsche et $a l^{23}$ found that algorithm-derived treatment recommendations were fully accepted by physicians in $85 \%$ of cases.

\section{Quantitative assessment of changes in pain intensity}

Pain was self-rated on a numerical rating out of 10 by intervention patients and controls at baseline and the end of the study in 15 trials (Post et $a l^{36}$ provided previously unpublished data to allow comparison of effect size in this review). Seven studies ${ }^{19} 2633364144$ rated pain using the Brief Pain Inventory, one ${ }^{24}$ used measures from the Amsterdam Pain Management Index, one study ${ }^{17}$ used the MD Anderson symptom inventory and one study ${ }^{45}$ used a validated $10 \mathrm{~cm}$ visual analogue scale. Five trials $\begin{array}{llllll}28 & 29 & 34 & 35 & 39 & \text { used }\end{array}$ simple non-validated numerical pain rating scales out of 10 points.

Forest plots summarising average pain intensity across 12 trials, and present pain across 3 trials are shown in figures 2 and 3 . Average pain refers to how a patient feels their pain has been overall and is a specific item in the Brief Pain Inventory. Studies which did not use the Brief Pain Inventory but provided a report of overall/cumulative pain severity as reported by the patient have been considered here under the heading of average pain intensity.

A statistically significant reduction in average pain intensity was found of around half a point out of 10 , mean difference -0.59 (95\% CI -0.87 to -0.30$)$. Removing the non-randomised study by Aubin et $a l^{19}$ from the meta-analysis did not significantly alter this result (mean difference -0.58 (95\% CI -0.90 to -0.26). The $\mathrm{I}^{2}$ statistic was $46 \%$ indicating moderate heterogeneity, which was expected in view of the heterogeneity of the interventions. One study by Mooney et $a l^{35}$ which had problems with fidelity appeared to be an outlier on the forest plot. A sensitivity analysis with this removed reduced the $\mathrm{I}^{2}$ statistic to $24 \%$.

Three studies reported 'present' pain intensity, that is, pain at the moment that it was being reported by the patient. There was no significant difference in present 


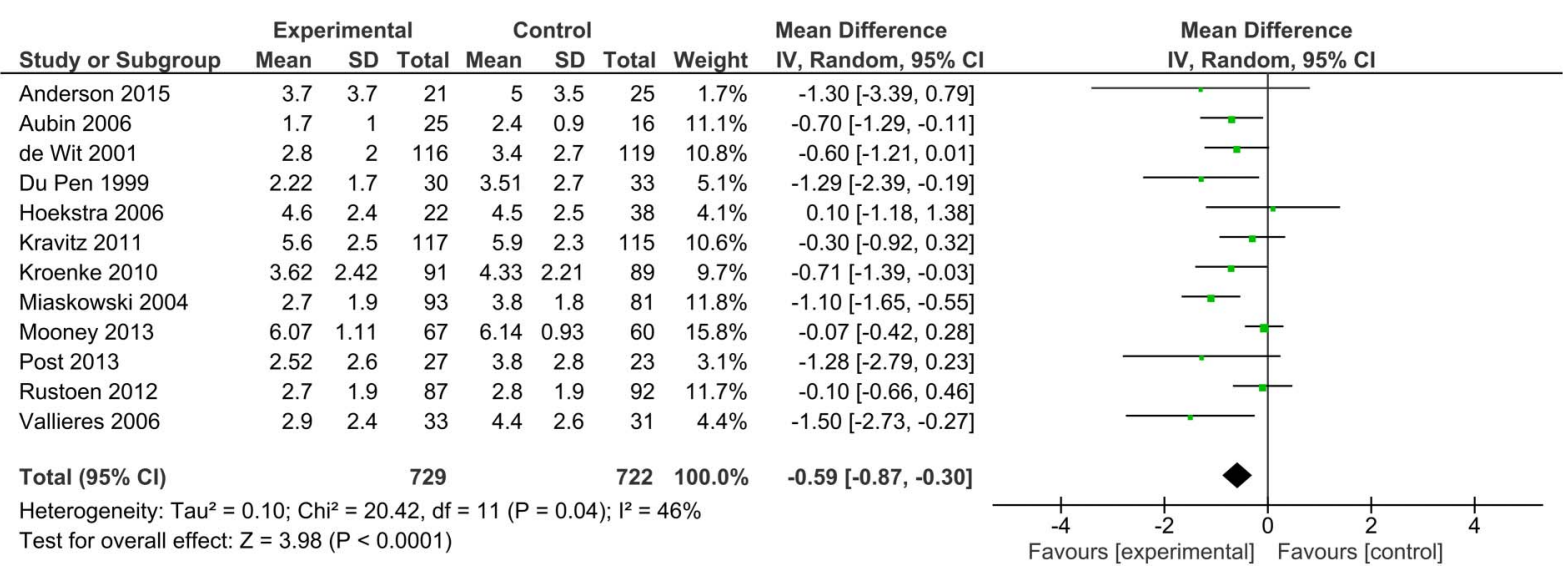

Figure 2 Forest plot of average/overall pain intensity.

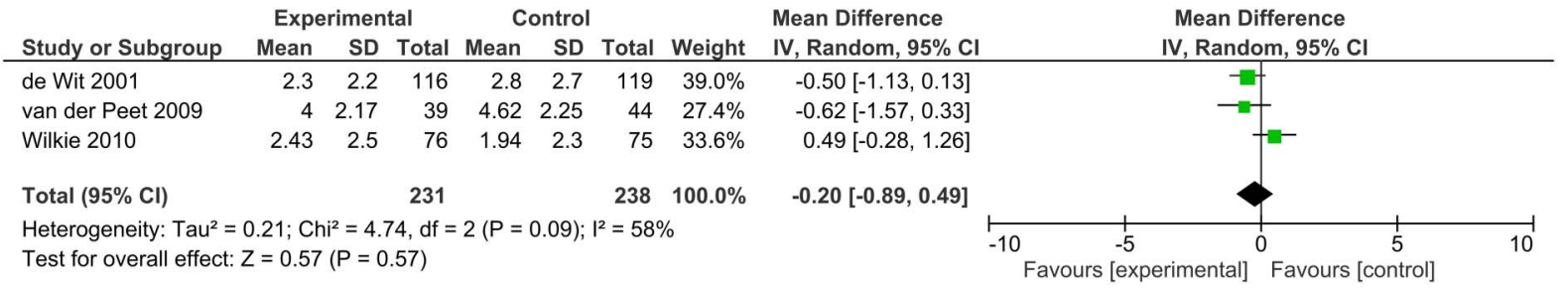

Figure 3 Forest plot of present pain intensity.

pain intensity between control and intervention groups, mean difference -0.20 ( $95 \% \mathrm{CI}-0.89$ to 0.49 ).

\section{Narrative summary of other pain-related outcomes}

Several studies included pain-related outcome measures other than pain intensity. Full details of the results of these outcome measures are included as an online supplementary table in appendix 2. Six studies (detailed in 10 reports) considered the effect of the PROMs on the clinical consultation. ${ }^{20} \quad 22 \quad 29-32 \quad 37 \quad 42-43 \quad 45$ Interventions were associated with more symptoms being reported and/or more discussions specifically about pain.

There was no evidence that opioid prescribing or the pain management index (an estimate of adequacy of analgesic prescription) was improved in the intervention groups compared with controls. ${ }^{17} 34394045$ However, one study by Bertsche $e t a l^{23}$ found significant improvements in guideline adherence over the intervention period.

Two studies ${ }^{17}{ }^{18}$ reported reductions in the number of pain threshold events over time in the intervention group compared with the control group, but these reductions only reached statistical significance in the study by Cleeland et al. ${ }^{18}$ The most frequent clinical response to pain threshold alerts in both studies ${ }^{17} 18$ was to reinforce existing management strategies.

\section{DISCUSSION}

\section{Main findings}

Feedback based on patient-reported pain outcomes has been used to effect changes in pain management in four main ways: (1) to provide reports about pain and additional symptoms to professionals (with the intention of increasing professional awareness of unrelieved pain and other problems); (2) to tailor patient pain education about self-management strategies and how to communicate about pain; (3) to prompt contact between a patient and professional when pain is above a set threshold; and (4) to link pain treatments to the severity of pain experienced by the patient via algorithmic management guidelines. Such interventions currently have a statistically significant but small effect $(<1$ point on a $0-10$ points rating scale) on patient-reported average pain intensity.

Previous reviews have shown that PROMs in oncology can improve patient satisfaction with care and consultation outcomes. This is the first review to have shown a significant impact of PROMs on a symptom outcome. However, it is accepted that for analgesics, patients desire reductions in pain of at least 50\%, ideally experiencing no worse than mild pain. ${ }^{47} \mathrm{~A}$ half-point improvement on a 10-point scale is not of such a magnitude. However, as monitoring pain and feeding this back to patients and/or professionals is fairly simple, the technique should be considered as part of more comprehensive programmes to tackle cancer pain.

The process evaluations described in three studies suggested that intervention fidelity was suboptimal, ${ }^{28} 3544$ which is likely to have reduced the effectiveness of interventions. Physicians failed to respond to symptom alerts and patients failed to take their data to consultations. Moreover, making professionals 
aware of high levels of patient-reported pain did not necessarily result in changes to analgesic prescribing. It is unclear from the evidence in this review as to why this might be the case. Previous studies have suggested that physicians can have a preference for their own judgement of symptoms over formal PROM measures. ${ }^{48}$ Another possibility is that numerical ratings of pain fail to take into consideration the complexity of pain experiences and individual patient preferences for pain management, which can become more apparent during the clinical consultation. Qualitative studies have shown that patients often manage pain around an acceptable level, and make trade-offs between opioid side effects, physical activity, cognitive function and pain relief. ${ }^{49}$ The interventions reviewed have not captured this complexity.

\section{Strengths and limitations}

This review was systematically conducted and identified trials spanning three decades. Twenty of the 22 trials included were RCTs and narrative description of these trials has allowed the components of interventions to be characterised. Despite the use of different measures of pain, we were able to obtain and combine pain data from 15 studies to allow for a meta-analysis of PROMs on clinically relevant outcomes. The main limitation of this review is that there were problems with intervention and trial description in several trials (table 3) which could have introduced bias. In addition, it is important to note that pain measurement was not the principal focus of every study included in this review. Some trials collected a range of symptoms and quality of life data including pain, and fed that back to patients and/or professionals. However, in all trials, pain was specifically monitored and pain-related outcomes were reported within the results, enabling comparison of pain data within this review.

\section{Implications for practice, policy and research}

Interventions which use PROMs to inform cancer pain management by patients and professionals show promise, but their usefulness and impact on pain might be enhanced if interventions are better designed and delivered. Based on the narrative review and considering the main components described by original study authors, we formulated a summary of the key steps that are necessary in order for these type of interventions to be effective (see figure 4). Arguably, a key component is the feedback process between patients and professionals and this requires further attention. The majority of studies in this review presented professionals with pain measures or threshold alerts without any instructions on how these measures should be used. This represents a missed opportunity since evidence-based cancer pain management guidelines exist to guide action.

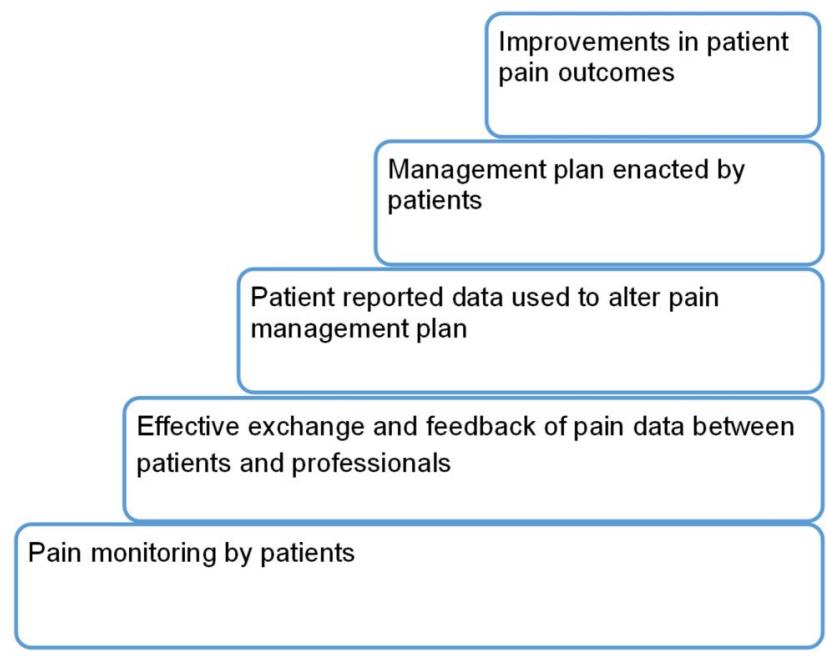

Figure 4 Steps by which PROM interventions can alter patient-reported pain intensity. PROM, patient-reported outcome measurement.

\section{CONCLUSIONS}

Interventions which have used patient-reported measurements to enhance the management of cancerrelated pain have achieved modest reductions in cancer pain intensity. The studies demonstrate that patients with cancer can provide their own data to guide management. The challenges are to provide effective transfer of information and to ensure clinicians act on this information in order to improve pain control.

Twitter Follow Rosalind Adam at @rosadamaberdeen

Contributors RA was involved in the design of this review, carried out database searches, assessed studies for inclusion in the review, performed data extraction, assessed risk of bias and was involved in the synthesis of results. CDB was involved in the design of this review, assessed studies for inclusion in the review, assessed risk of bias of included studies, and contributed to drafting and revising the article critically. CMB was involved in the design of this review, assessed studies for inclusion in the review, and contributed to drafting and revising the article critically. MdB was involved in the design of this review, assessed studies for inclusion in the review, and contributed to drafting and revising the article critically. PM was involved in the design of this review, assessed studies for inclusion in the review, and contributed to drafting and revising the article critically.

Funding RA completed this review during a clinical academic fellowship funded by the Chief Scientist Office of the Scottish Government, grant reference RG12141-10.

Competing interests None declared.

Provenance and peer review Not commissioned; externally peer reviewed.

Open Access This is an Open Access article distributed in accordance with the Creative Commons Attribution Non Commercial (CC BY-NC 4.0) license, which permits others to distribute, remix, adapt, build upon this work non-

commercially, and license their derivative works on different terms, provided the original work is properly cited and the use is non-commercial. See: http://creativecommons.org/licenses/by$\mathrm{nc} / 4.0 /$ 


\section{REFERENCES}

1 van den Beuken-van Everdingen MH, de Rijke JM, Kessels AG, et al. Prevalence of pain in patients with cancer: a systematic review of the past 40 years. Ann Oncol 2007;18:1437-49.

2 Breivik H, Cherny N, Collett B, et al. Cancer-related pain: a pan-European survey of prevalence, treatment, and patient attitudes. Ann Oncol 2009;20:1420-33.

3 Oldenmenger WH, Sillevis Smitt PA, van Dooren S, et al. A systematic review on barriers hindering adequate cancer pain management and interventions to reduce them: a critical appraisal. Eur J Cancer 2009;45:1370-80.

4 Adam R, Bond C, Murchie P. Educational interventions for cancer pain. A systematic review of systematic reviews with nested narrative review of randomized controlled trials. Patient Educ Couns 2015;98:269-82.

5 Shi Q, Wang XS, Mendoza TR, et al. Assessing persistent cancer pain: a comparison of current pain ratings and pain recalled from the past week. J Pain Symptom Manage 2009;37:168-74.

6 Cleeland CS. Barriers to the management of cancer pain. Oncology (Williston Park, NY) 1987;1(Suppl 2):19-26.

7 Adam R, Clausen MG, Hall S, et al. Utilising out-of-hours primary care for assistance with cancer pain: a semi-structured interview study of patient and caregiver experiences. $\mathrm{Br} \mathrm{J} \mathrm{Gen}$ Pract 2015;65:e754-60.

8 Elliott TE, Murray DM, Oken MM, et al. Improving cancer pain management in communities: main results from a randomized controlled trial. J Pain Symptom Manage 1997;13:191-203.

9 Dawson J, Doll H, Fitzpatrick R, et al. The routine use of patient reported outcome measures in healthcare settings. BMJ 2010;340:c186.

10 US Department of Health and Human Services Food and Drug Administration. Guidance for industry: patient-reported outcome measures: use in medical product development to support labeling claims. 2009. http://www.fda.gov/downloads/ Drugs/GuidanceComplianceRegulatoryInformation/Guidances/ UCM193282.pdf (accessed 29 Feb 2016).

11 Greenhalgh J. The applications of PROs in clinical practice: what are they, do they work, and why? Qual Life Res 2009;18:115-23.

12 Chen J, Ou L, Hollis SJ. A systematic review of the impact of routine collection of patient reported outcome measures on patients, providers and health organisations in an oncologic setting. BMC Health Serv Res 2013;13:211.

13 Kotronoulas G, Kearney N, Maguire R, et al. What is the value of the routine use of patient-reported outcome measures toward improvement of patient outcomes, processes of care, and health service outcomes in cancer care? A systematic review of controlled trials. J Clin Oncol 2014;32:1480-501.

14 Higgins JP, Altman DG, Gøtzsche PC, et al. The Cochrane collaboration's tool for assessing risk of bias in randomised trials. BMJ 2011;343:d5928.

15 Cohen J. A coefficient of agreement for nominal scales. Educ Psychol Meas 1960;20:37-46.

16 Hoffmann TC, Glasziou PP, Boutron I, et al. Better reporting of interventions: template for intervention description and replication (TIDieR) checklist and guide. BMJ 2014;348:g1687.

17 Anderson KO, Palos GR, Mendoza TR, et al. Automated pain intervention for underserved minority women with breast cancer. Cancer 2015;121:1882-90.

18 Cleeland CS, Wang XS, Shi Q, et al. Automated symptom alerts reduce postoperative symptom severity after cancer surgery: a randomized controlled clinical trial. J Clin Oncol 2011;29:994-1000.

19 Aubin M, Vézina L, Parent R, et al. Impact of an educational program on pain management in patients with cancer living at home. Oncol Nurs Forum 2006;33:1183-8.

20 Berry DL, Blumenstein BA, Halpenny B, et al. Enhancing patient-provider communication with the electronic self-report assessment for cancer: a randomized trial. J Clin Oncol 2011;29:1029-35.

21 Berry DL, Hong F, Halpenny B, et al. Electronic self-report assessment for cancer and self-care support: results of a multicenter randomized trial. J Clin Oncol 2014;32:199-205.

22 Berry DL, Hong F, Halpenny B, et al. The electronic self report assessment and intervention for cancer: promoting patient verbal reporting of symptom and quality of life issues in a randomized controlled trial. BMC Cancer 2014;14:513.

23 Bertsche T, Askoxylakis V, Habl G, et al. Multidisciplinary pain management based on a computerized clinical decision support system in cancer pain patients. Pain 2009;147:20-8.

24 de Wit R, van Dam F, Zandbelt L, et al. A pain education program for chronic cancer pain patients: follow-up results from a randomized controlled trial. Pain 1997;73:55-69.

25 de Wit R, van Dam F, Loonstra S, et al. Improving the quality of pain treatment by a tailored pain education programme for cancer patients in chronic pain. Eur J Pain 2001;5:241-56.

26 Du Pen SL, Du Pen AR, Polissar N, et al. Implementing guidelines for cancer pain management: results of a randomized controlled clinical trial. J Clin Oncol 1999;17:361-70.

27 Given C, Given B, Rahbar M, et al. Effect of a cognitive behavioral intervention on reducing symptom severity during chemotherapy. J Clin Oncol 2004;22:507-16.

28 Hoekstra J, de Vos R, van Duijn NP, et al. Using the symptom monitor in a randomized controlled trial: the effect on symptom prevalence and severity. J Pain Symptom Manage 2006;31:22-30.

29 Kravitz RL, Tancredi DJ, Grennan T, et al. Cancer Health Empowerment for Living without Pain (Ca-HELP): effects of a tailored education and coaching intervention on pain and impairment. Pain 2011;152:1572-82.

30 Kravitz RL, Tancredi DJ, Jerant A, et al. Influence of patient coaching on analgesic treatment adjustment: secondary analysis of a randomized controlled trial. J Pain Symptom Manage 2012;43:874-84.

31 Street RL Jr, Slee C, Kalauokalani DK, et al. Improving physician-patient communication about cancer pain with a tailored education-coaching intervention. Patient Educ Couns 2010;80:42-7.

32 Street RL Jr, Tancredi DJ, Slee C, et al. A pathway linking patient participation in cancer consultations to pain control. Psychooncology 2014;23:1111-17.

33 Kroenke K, Theobald D, Wu J, et al. Effect of telecare management on pain and depression in patients with cancer: a randomized trial. JAMA 2010;304:163-71.

34 Miaskowski C, Dodd M, West C, et al. Randomized clinical trial of the effectiveness of a self-care intervention to improve cancer pain management. J Clin Oncol 2004;22:1713-20.

35 Mooney KH, Beck SL, Friedman RH, et al. Automated monitoring of symptoms during ambulatory chemotherapy and oncology providers' use of the information: a randomized controlled clinical trial. Support Care Cancer 2014;22:2343-50. 
36 Post DM, Shapiro CL, Cegala DJ, et al. Improving symptom communication through personal digital assistants: the CHAT (Communicating Health Assisted by Technology) project. J Natl Cancer Inst Monographs 2013;2013:153-61.

37 Ruland CM, Holte HH, Røislien J, et al. Effects of a computer-supported interactive tailored patient assessment tool on patient care, symptom distress, and patients' need for symptom management support: a randomized clinical trial. J Am Med Inform Assoc 2010;17:403-10.

38 Rustøen T, Valeberg BT, Kolstad E, et al. The PRO-SELF((c)) Pain Control Program improves patients' knowledge of cancer pain management. J Pain Symptom Manage 2012;44:321-30.

39 Rustøen T, Valeberg BT, Kolstad E, et al. A randomized clinical trial of the efficacy of a self-care intervention to improve cancer pain management. Cancer Nurs 2014;37:34-43.

40 Trowbridge R, Dugan W, Jay SJ, et al. Determining the effectiveness of a clinical-practice intervention in improving the control of pain in outpatients with cancer. Acad Med 1997;72:798-800.

41 Vallières I, Aubin M, Blondeau L, et al. Effectiveness of a clinical intervention in improving pain control in outpatients with cancer treated by radiation therapy. Int J Radiat Oncol Biol Phys 2006;66:234-7.

42 Velikova G, Booth L, Smith AB, et al. Measuring quality of life in routine oncology practice improves communication and patient well-being: a randomized controlled trial. J Clin Oncol 2004;22:714-24.

43 Takeuchi EE, Keding A, Awad N, et al. Impact of patient-reported outcomes in oncology: a longitudinal analysis of patient-physician communication. J Clin Oncol 2011;29:2910-17.

44 van der Peet EH, van den Beuken-van Everdingen MH, Patijn $\mathrm{J}$, et al. Randomized clinical trial of an intensive nursing-based pain education program for cancer outpatients suffering from pain. Support Care Cancer 2009;17:1089-99.

45 Wilkie D, Berry D, Cain K, et al. Effects of coaching patients with lung cancer to report cancer pain. West J Nurs Res 2010;32:23-46.

46 Johnson D. The behavioral system model for nursing. In: Roy J, ed. Conceptual methods for nursing practice. Vol 1. ed. New York: Appleton-Century-Crofts, 1980:205-16.

47 Moore RA, Straube S, Aldington D. Pain measures and cut-offs - 'no worse than mild pain' as a simple, universal outcome. Anaesthesia 2013;68:400-12.

48 Cox A, Illsley M, Knibb W, et al. The acceptability of e-technology to monitor and assess patient symptoms following palliative radiotherapy for lung cancer. Palliat Med 2011;25:675-81.

49 Manzano A, Ziegler L, Bennett M. Exploring interference from analgesia in patients with cancer pain: a longitudinal qualitative study. J Clin Nurs 2014;23:1877-88. 